\title{
A modified Mini Nutritional Assessment without BMI can effectively assess the nutritional status of neuropsychiatric patients
}

\author{
Alan C Tsai, Yuan-Ti Chou, Tsui-Lan Chang, Shu-Nu Chang-Lee and Shwu-Feng Tsay
}

\begin{abstract}
Aim and objectives. To determine whether a modified version of the Mini Nutritional Assessment (MNA) without body mass index (BMI) can effectively identify individuals at risk of malnutrition among patients with neuropsychiatric disorders.

Background. Neuropsychiatric patients have an additional risk of nutritional disorder due to functional impairments and drug effects. However, their nutritional status is generally neglected. It is important to find a tool that is simple, easy to use and noninvasive.

Design. The study involved 105 patients in the acute phase of confirmed neuropsychiatric disorders in an area hospital. All subjects were cognitively able to have effective verbal communication.

Method. The study included serum biochemical and anthropometric measurements and an on-site, in-person interview using a structured questionnaire to elicit personal data, health condition and answers to questions in the MNA. Subjects' nutritional statuses were graded with a MNA that adopted population-specific anthropometric cut-off points or one further with the BMI question removed and its assigned score redistributed to other anthropometric questions.

Results. Both versions of the modified MNA effectively graded the nutritional status of neuropsychiatric patients and showed good correlations with the major nutritional indicators such as BMI, calf circumference and the length of hospital stay.

Conclusions. The MNA can effectively assess the nutritional status of neuropsychiatric patients and enhance timely detection and intervention of their nutritional disorders. A modified MNA without the BMI question can maintain the full functionality of the tool. The version does not require weight and height measurements and thus will enhance the usefulness of the instrument. Relevance to clinical practice. Neuropsychiatric patients are a high-risk group of nutritional disorders. The MNA, especially the one without BMI, has the potential to improve professional efficiency of the primary care workers.
\end{abstract}

Key words: assessment, mental health, nurses, nursing, nutrition, older people

Accepted for publication: 4 September 2008

\section{Introduction}

Approximately $14 \%$ of the global burden of disease has been attributed to neuropsychiatric disorders. Thus, the delivery of mental health care can greatly affect the outcome of health care (Prince et al. 2007). People with neuropsychiatric problems have an additional risk of nutritional disorder due to factors such as medication, drug-nutrient interaction, poor self-care, inability to shop or prepare foods, delusions and social isolation (Sullivan et al. 1999). Unhealthy lifestyle such as poor diet, lack of exercise, smoking and substance abuse are common among neuropsychiatric patients (Brown
Authors: Alan C Tsai, PhD, Department of Environmental Health Sciences, School of Public Health, University of Michigan, Ann Arbor, MI, USA and Department of Healthcare Administration, Asia University, Wu-Feng, Taichung, Taiwan; Yuan-Ti Chou, MS, Department of Healthcare Administration, Asia University, WuFeng, Taichung, Taiwan; Tsui-Lan Chang, MS, Department of Healthcare Administration, Asia University, Wu-Feng, Taichung,
Taiwan, China; Shu-Nu Chang-Lee, PhD, Department of Healthcare Administration, Asia University, Wu-Feng, Taichung, Taiwan; Shwu-Feng Tsay, PhD, Department of Health Services Management, China Medical University, Taichung, Taiwan Correspondence: Alan C Tsai, PhD, 3411 E. Dobson Place, Ann Arbor, MI 48105, USA. Telephone: +1 7347612468 .

E-mail: atsai@umich.edu 
et al. 1999). Weight changes, behavioural problems and eating disorders are common in neuropsychiatric patients. Patients with schizophrenia are often associated with obesity or overweight, while patients with depression are often associated with depressed appetite and weight loss (Gray \& Gray 1989, McCreadie 2003). Asplund et al. (1981) observed that $30 \%$ of psychogeriatric patients had energy or protein-energy malnutrition and $4 \%$ had obesity. Molteno et al. (2000) observed that $32 \%$ of males and $26 \%$ of females with mental handicap were underweight whereas $6 \%$ of males and $17 \%$ of females were obese. Recent studies have also found that the intakes of micro nutrients such as the $\mathrm{B}$ vitamins and omega-3 fatty acids play a role in mental health (Coppen \& Bolander-Gouaille 2005, Kerperman et al. 2006, Gariballa \& Forster 2007). Of course, individuals with neuropsychiatric disorders, like the general population, are also confronted with the common lifestyle-related diseases such as hypertension, diabetes, heart disease and cancer. Despite this, neuropsychiatric patients appear to be a neglected group. Well-planned nutritional assessments rarely take place in psychiatric hospitals. Many psychiatric centres rely on nurses' subjective judgment or simple indicators such as serum protein concentrations for making nutrition-related decisions. However, these judgments are not always reliable or adequate. For example, Abayomi and Hackett (2004) observed that nurses overlooked $29 \%$ of nutritionally at risk patients. Thus, a well-planned nutritional assessment should be an integral component of a comprehensive psychiatric assessment (Saxena et al. 2006). A well-planned nutritional assessment is crucial, because progressive undernutrition can often go undetected. The prevention of undernutrition requires the identification of the causes to adopt corrective actions for optimal nutritional intervention (Guigoz et al. 2002). We have recently modified the Mini Nutritional Assessment (MNA) (Guigoz et al. 1994) by adopting the population-specific anthropometric cut-points and found that the modified tool has improved functionality in predicting the nutritional status of Taiwanese older people living in the community or in various care facilities (Tsai et al. 2007a, Tsai \& Ku 2008, Tsai \& Shih 2009). We also have observed that a further modified version that had the body mass index (BMI) question removed from the scale functioned equally well as one that included the BMI (Tsai et al. 2009). Although the MNA was developed and validated with older adults, we thought it would be of interest to determine whether this tool, especially the version without BMI, would be useful for identifying individuals at risk of malnutrition among patients with neuropsychiatric disorders who may generally be of younger age. The noninvasive nature and its ease of use coupled with the lack of a more specific and readily available tool make it a worthwhile attempt.

\section{Method}

Using patients residing in psychiatric wards at a psychiatric hospital in central Taiwan as a convenient sample, this study evaluated the nutritional status of those patients with two modified versions of the MNA. Patients who had been in the acute phase of confirmed neuropsychiatric disorders for three months or longer and judged cognitively able to have effective verbal communication were recruited to participate in the study. Patients who were diagnosed as having personality, adjustment or developmental disorders according to the Diagnostic and Statistical Manual of Mental Disorders (DSM-IV-TR) (American Psychiatric Association 2000) were excluded from the study. Eligible subjects or their legal guardians were explained of the objective and process of the study. A total of 105 patients (67 men and 38 women) met those conditions and agreed to participate in the study. The protocol and ethical aspect of the study was approved by the Institutional Review Board of the Hospital. Written consents were obtained from the legal guardians and subjects' confidentiality was maintained throughout the study.

The study included three components: (1) measurements of fasting blood biochemical indicators including serum albumin, haemoglobin, haematocrit, glucose, serum creatinine, triglyceride and total cholesterol as a part of hospital's routine laboratory tests, (2) measurements of anthropometric indicators including body weight, height and waist, hip, midarm and calf circumferences according to standard methods (Lee \& Nieman 2003b), and (3) an on-site, in-person, interview with a structured questionnaire for eliciting sociodemographic and health-related personal data, appetite and mood statuses and answers to questions in the MNA (Guigoz et al. 1994, Tsai et al. 2009). The caregivers were allowed to assist patients answering the questions or to provide the information on their behalf, if necessary. Anthropometric measurements and the questionnaire interview were carried out within one week of the blood sampling (for biochemical measurements) date. All interviews and physical measurements were carried out by the same (one) interviewer accompanied by the caregivers.

Nutritional status of each subject was assessed with two modified versions of the MNA: the MNA-TI (MNA-Taiwan version I) which adopted Taiwanese-specific anthropometric (BMI, mid-arm circumference, MAC; calf circumference, CC) cut-points and the MNA-TII (MNA-Taiwan version II) which eliminated the BMI question (Question F) from the MNA-TI and reassigned one point of the three BMI points to the MAC 
question (Question Q) and two to the CC question (Question R) (Tsai et al. 2009). All other questions were the same for both versions and were unchanged from the original MNA (Guigoz et al. 1994).

Data were analysed with sPSs/Windows 10.0 software package. Simple statistics was expressed as mean \pm SD. Wilcoxon signed-rank test was performed to determine the significance of differences between the patterns of nutritional status predicted by the two modified versions. Pearson's correlation analyses were conducted to determine the level of correlation of the anthropometric, biochemical and healthrelated parameters with the total MNA scores and also with the concentration of serum albumin.

\section{Results}

Table 1 shows the sociodemographic, lifestyle- and healthrelated statuses of study subjects. Fifty-eight per cent of patients were 45 years or younger. Only five subjects $(4.8 \%$, all female) were over 65 years old. Nearly $50 \%$ of patients were never married. Approximately $50 \%$ of all subjects had less than nine years of formal education and the rest had more. About two-thirds of patients lived with family members and $19 \%$ in care institutions. According to DSM-IV, approximately $50 \%$ of subjects were classified schizophrenia, $21 \%$ major depression, $15 \%$ bipolar disorder and $14.3 \%$ other types. Twenty-seven percent of subjects had the disorder for less than five years, another 27\% 5-10 years and the rest 10 years or more. Forty-five per cent of subjects had other chronic diseases in addition to neuropsychiatric disorders. Thirty-eight per cent of subjects indicated good or very good appetite, $42 \%$ neutral and $16 \%$ poor or very poor appetite. Thirteen percent of subjects lost more than $3 \%$ of body weight during the last three months.

Table 2 shows the anthropometrical and biochemical characteristics of the patients. The average BMI was $24.9 \mathrm{~kg} / \mathrm{m}^{2}$ for male and $24.0 \mathrm{~kg} / \mathrm{m}^{2}$ for female patients. The average MAC was 28.4 and $26.4 \mathrm{~cm}$ and the average CC was 34.8 and $33.2 \mathrm{~cm}$ for male and female patients, respectively. Average waist circumferences were 87.7 and $80.5 \mathrm{~cm}$ and average hip circumferences were 95.7 and $96.7 \mathrm{~cm}$ for male and female patients, respectively. Average haemoglobin concentrations were 14.5 and $12.9 \mathrm{~g} / \mathrm{dl}$, albumin, $4 \cdot 1$ and $4 \cdot 1$, triglyceride, 152 and $135 \mathrm{mg} / \mathrm{dl}$, cholesterol, 170 and $177 \mathrm{mg} / \mathrm{dl}$, fasting serum glucose, 97 and $102 \mathrm{mg} / \mathrm{dl}$, and serum creatinine, 0.8 and $0.7 \mathrm{mg} / \mathrm{dl}$ for male and female patients, respectively.

Subjects' scoring patterns of the MNA questions are shown in Table 3. Subjects scored poorly on several questions. Approximately $27 \%$ of patients had reduced food intake
Table 1 Characteristics of subjects $(n, \%)$

\begin{tabular}{|c|c|c|c|}
\hline Item & $\begin{array}{l}\text { Men } \\
(n=67)\end{array}$ & $\begin{array}{l}\text { Women } \\
(n=38)\end{array}$ & All \\
\hline \multicolumn{4}{|l|}{ Age (years) } \\
\hline$<45$ & $45(67 \cdot 2)$ & $16(42 \cdot 1)$ & $61(58 \cdot 1)$ \\
\hline $45-64$ & $22(32 \cdot 8)$ & $17(44 \cdot 8)$ & $39(37 \cdot 1)$ \\
\hline$\geq 65$ & 0 & $5(13 \cdot 1)$ & $5(4 \cdot 8)$ \\
\hline \multicolumn{4}{|l|}{ Marital status } \\
\hline Never married & $41(61 \cdot 2)$ & $11(28 \cdot 9)$ & $52(49 \cdot 5)$ \\
\hline Married & $15(22 \cdot 4)$ & $14(36 \cdot 9)$ & $29(27 \cdot 6)$ \\
\hline Divorced/widowed & $11(16 \cdot 4)$ & $13(34 \cdot 2)$ & $24(22 \cdot 9)$ \\
\hline \multicolumn{4}{|c|}{ Years of formal education } \\
\hline$\leq 6$ & $15(22 \cdot 4)$ & $13(34 \cdot 1)$ & $28(26 \cdot 7)$ \\
\hline $7-9$ & $16(23 \cdot 9)$ & $8(21 \cdot 1)$ & $24(22 \cdot 9)$ \\
\hline $10-12$ & $29(43 \cdot 3)$ & $10(26 \cdot 3)$ & $39(37 \cdot 1)$ \\
\hline$>12$ & $7(10 \cdot 4)$ & $7(18 \cdot 5)$ & $14(13 \cdot 3)$ \\
\hline \multicolumn{4}{|l|}{ Living status } \\
\hline Alone & $5(7 \cdot 5)$ & $3(7 \cdot 9)$ & $8(7 \cdot 6)$ \\
\hline With family & $40(59 \cdot 7)$ & $28(73 \cdot 7)$ & $68(64 \cdot 8)$ \\
\hline Care institutions & $18(26 \cdot 8)$ & $2(5 \cdot 2)$ & $20(19 \cdot 0)$ \\
\hline Other & $4(6 \cdot 0)$ & $5(13 \cdot 2)$ & $9(8 \cdot 6)$ \\
\hline \multicolumn{4}{|l|}{ Employment } \\
\hline No & $57(85 \cdot 1)$ & $35(92 \cdot 1)$ & $92(87 \cdot 6)$ \\
\hline Yes & $10(14 \cdot 9)$ & $3(7 \cdot 9)$ & $13(9 \cdot 5)$ \\
\hline \multicolumn{4}{|c|}{ Diagnosed mental disorder* } \\
\hline Schizophrenia & $35(52 \cdot 2)$ & $17(44 \cdot 7)$ & $52(49 \cdot 5)$ \\
\hline Major depression & $16(23 \cdot 9)$ & $6(15 \cdot 8)$ & $22(21 \cdot 0)$ \\
\hline Bipolar disorder & $10(14 \cdot 9)$ & $6(15 \cdot 8)$ & $16(15 \cdot 2)$ \\
\hline Other & $6(9 \cdot 0)$ & $9(23 \cdot 7)$ & $15(14 \cdot 3)$ \\
\hline \multicolumn{4}{|c|}{ Mental disorder history (years) } \\
\hline$<5$ & $12(17 \cdot 9)$ & $16(42 \cdot 0)$ & $28(26 \cdot 7)$ \\
\hline $5-10$ & $21(31 \cdot 3)$ & $7(18 \cdot 5)$ & $28(26 \cdot 7)$ \\
\hline$>10$ & $34(50 \cdot 7)$ & $15(39 \cdot 5)$ & $49(46 \cdot 7)$ \\
\hline \multicolumn{4}{|l|}{ Other chronic diseases } \\
\hline No & $36(53 \cdot 7)$ & $22(57 \cdot 9)$ & $58(55 \cdot 2)$ \\
\hline Yes & $31(46 \cdot 3)$ & $16(42 \cdot 1)$ & $47(44 \cdot 8)$ \\
\hline \multicolumn{4}{|l|}{ Appetite status } \\
\hline Very good & $9(13 \cdot 4)$ & $4(10 \cdot 5)$ & $13(12 \cdot 3)$ \\
\hline Good & $22(32 \cdot 8)$ & $9(23.7)$ & $31(29 \cdot 5)$ \\
\hline Neutral & $26(38 \cdot 8)$ & $18(47 \cdot 4)$ & $44(41 \cdot 9)$ \\
\hline Poor & $10(14 \cdot 9)$ & $5(13 \cdot 2)$ & $15(14 \cdot 3)$ \\
\hline Very poor & $0(0)$ & $2(5 \cdot 3)$ & $2(1.9)$ \\
\hline \multicolumn{4}{|c|}{$\%$ Weight loss during last three months } \\
\hline$>5$ & $2(3 \cdot 0)$ & $2(5 \cdot 3)$ & $4(3 \cdot 8)$ \\
\hline $3-5$ & $7(10 \cdot 4)$ & $3(7 \cdot 9)$ & $10(9 \cdot 5)$ \\
\hline $2-3$ & $5(7 \cdot 5)$ & $4(10 \cdot 5)$ & $9(8 \cdot 6)$ \\
\hline$\leq 2$ & $53(79 \cdot 1)$ & $29(76 \cdot 3)$ & $82(78 \cdot 1)$ \\
\hline
\end{tabular}

*According to DSM-IV classifications.

during the past three months and a similar proportion $(25 \%)$ of subjects had reduced weight during the same period. Twenty percent had psychological stress and $24 \%$ had dementia or depression. More than half $(54 \%)$ of patients were on four or more kinds of prescribed medicine while $37 \%$ had inadequate vegetable or fruit intake and $57 \%$ had inadequate water intake. Approximately $45 \%$ of subject 
Table 2 Anthropometrical and biochemical measurements (Mean \pm $\mathrm{SD})$ of subjects

\begin{tabular}{lcr}
\hline & $\begin{array}{l}\text { Men } \\
(n=67)\end{array}$ & $\begin{array}{c}\text { Women } \\
(n=38)\end{array}$ \\
\hline Item & $167 \cdot 2 \pm 6 \cdot 9$ & $155 \cdot 6 \pm 5 \cdot 8$ \\
Body height $(\mathrm{cm})$ & $69 \cdot 8 \pm 13 \cdot 1$ & $58 \cdot 4 \pm 13 \cdot 8$ \\
Body weight $(\mathrm{kg})$ & $24 \cdot 9 \pm 4 \cdot 3$ & $24 \cdot 0 \pm 5 \cdot 1$ \\
BMI $\left(\mathrm{kg} / \mathrm{m}^{2}\right)$ & $28 \cdot 4 \pm 3 \cdot 0$ & $26 \cdot 4 \pm 4 \cdot 2$ \\
MAC $(\mathrm{cm})$ & $34 \cdot 8 \pm 3 \cdot 6$ & $33 \cdot 2 \pm 5 \cdot 0$ \\
CC $(\mathrm{cm})$ & $87 \cdot 7 \pm 11 \cdot 3$ & $80 \cdot 5 \pm 11 \cdot 4$ \\
Waist circumference $(\mathrm{cm})$ & $95 \cdot 7 \pm 8 \cdot 0$ & $96 \cdot 7 \pm 10 \cdot 7$ \\
Hip circumference $(\mathrm{cm})$ & $14 \cdot 5 \pm 1 \cdot 3$ & $12 \cdot 9 \pm 1 \cdot 4$ \\
Haemoglobin $(\mathrm{g} / \mathrm{dl})$ & $41 \cdot 0 \pm 5 \cdot 9$ & $37 \cdot 5 \pm 4 \cdot 1$ \\
Haematocrit $(\%)$ & $4 \cdot 1 \pm 0 \cdot 4$ & $4 \cdot 1 \pm 0 \cdot 3$ \\
Serum albumin $(\mathrm{g} / \mathrm{dl}) *$ & $6 \cdot 9 \pm 0 \cdot 6$ & $7 \cdot 0 \pm 0 \cdot 7$ \\
Total serum protein $(\mathrm{g} / \mathrm{dl})$ & $152 \pm 99$ & $135 \pm 59$ \\
Serum triglyceride $(\mathrm{mg} / \mathrm{dl})$ & $170 \pm 45$ & $177 \pm 42$ \\
Serum cholesterol $(\mathrm{mg} / \mathrm{dl})$ & $96 \cdot 5 \pm 27 \cdot 9$ & $102 \cdot 4 \pm 43 \cdot 2$ \\
Fasting serum glucose $(\mathrm{mg} / \mathrm{dl})$ & $10 \cdot 2 \pm 4 \cdot 3$ & $12 \cdot 2 \pm 5 \cdot 2$ \\
Serum urea N $(\mathrm{mg} / \mathrm{dl})$ & $0 \cdot 8 \pm 0 \cdot 3$ & $0 \cdot 7 \pm 0 \cdot 4$ \\
Serum creatinine $(\mathrm{mg} / \mathrm{dl})$ & $6 \cdot 6 \pm 1 \cdot 7$ & $5 \cdot 7 \pm 1 \cdot 5$ \\
Serum uric acid $(\mathrm{mg} / \mathrm{dl})$ & $51 \cdot 2 \pm 72 \cdot 0$ & $28 \cdot 0 \pm 22 \cdot 9$ \\
SGOT $(\mathrm{U} / \mathrm{l})$ & $43 \cdot 4 \pm 45 \cdot 5$ & $28 \cdot 6 \pm 23 \cdot 2$ \\
SGPT (U/l) & &
\end{tabular}

BMI, body mass index; MAC, mid-arm circumference; CC, calf circumference; SGOT, serum glutamate-oxaloacetate transaminase; SGPT, serum glutamate-pyruvate transaminase.

*Six men $(9.0 \%)$ and 2 women's $(5.3 \%)$ serum albumin concentrations were $<3.5(\mathrm{~g} / \mathrm{dl})$.

thought they were malnourished or were unsure about their nutritional status while $55 \%$ thought they had poor health relative to their peers or were unsure about their health status.

The distributions of nutritional statuses assessed according to the two versions of the MNA are shown in Table 4. According to MNA-TI, 7.6, 21.9 and $70.5 \%$ of patients were predicted malnourished, at risk of malnutrition and normal, respectively. The respective values were 7.6, 20 and $72 \cdot 4 \%$, respectively, for MNA-TII. Analysis of these distributions with Wilcoxon signed-rank test showed that no significant differences exist between these two distribution patterns.

Pearson's correlation coefficients $(r)$ of the total MNA scores or serum albumin concentrations with each of the major nutrition- or health-related parameters are shown in Table 5. The total MNA scores of both versions were very highly significantly correlated with subjects' BMI, MAC (in men), CC (in men), number of prescribed drugs, self-assessed appetite status and \% weight loss (all $p<0 \cdot 001$ ). Total MNA scores were also significantly correlated with serum albumin concentrations $(p<0.05)$, CC (in women, $p<0.01$ ) and MAC (in women, $p<0.05$ ). Both versions of the MNA had comparable $r$ values. All parameters had weaker correlations with serum albumin than with the MNA scores.

\section{Discussion}

\section{Functionality of the modified MNA}

Both versions of the modified MNA predicted 8 patients $(7 \cdot 6 \%)$ malnourished. The MNA-TI further predicted 23 patients $(21.9 \%)$ and the MNA-TII further predicted 21 patients $(20 \%)$ at risk of malnutrition. Analysis of these results with the Wilcoxon signed-rank test suggests that no differences in the patterns predicted by these two versions of the MNA. All 29 patients predicted malnourished or at risk of malnutrition with the MNA-TII were among the 31 predicted with the MNA-TI. Pearson's correlation analyses showed that both versions of the modified MNA predicted nutritional statuses that have comparable degrees of correlations with each of the key nutritional indicators including serum albumin, BMI, MAC, CC, number of prescribed drugs, self-assessed appetite status and \% weight loss. These results suggest that both versions of the modified MNA can function equally well and the MNA-TII which has the BMI question (Question F) eliminated from the screen can function as effectively as the MNA-TI in predicting the nutritional risk status of the neuropsychiatric patients.

The observed rates of malnutrition $(7 \cdot 6 \%)$ and at risk of malnutrition (20-22\%) are in concordance with the expected high prevalence of malnutrition among neuropsychiatric patients. The rates are higher than the $1.5 \%$ malnourished and $10.7 \%$ at risk of malnutrition observed in the general population of Taiwanese, 65 years or older (Tsai et al. $2007 \mathrm{a}, \mathrm{b}$ ), or the $0 \cdot 7 \%$ malnourished and $10-12 \%$ at risk of malnutrition in community-living older people (Tsai et al. unpublished observation). However, these rates are relatively low compared to the $24 \%$ malnourished and $57 \%$ at risk of malnutrition of stroke rehabilitation patients (Tsai \& Shih 2009), or $14-20 \%$ malnourished and $60 \%$ at risk of malnutrition of institutionalised older people observed in our recent studies (Tsai \& Ku 2008).

There are relatively few studies assessing the nutritional status of neuropsychiatric patients. Asplund et al. (1981) assessed nutritional status of patients in psychogeriatric wards based on anthropometric parameters and circulating proteins and found that neuropsychiatric patients had low mean values on these parameters and $30 \%$ of patients were undernourished. Molteno et al. (2000) examined nutritional status of mental patients in a long-stay hospital and found that $32 \%$ of males and $26 \%$ of females had BMI $<20$. Abayomi and Hackett (2004) compared nurses' judgment vs. a nutrition risk tool on neuropsychiatric patients' nutritional status and found that nurses overlooked $29 \%$ at risk patients. 
Table 3 Subjects' scoring patterns of each of the MNA questions $(n=105)$

\begin{tabular}{|c|c|c|}
\hline Question & Score & $\%$ \\
\hline \multicolumn{3}{|l|}{ Food intake declined over the past 3 months } \\
\hline Severe & 0 & $1 \cdot 9$ \\
\hline Moderate & 1 & $24 \cdot 8$ \\
\hline No decline & 2 & $73 \cdot 3$ \\
\hline \multicolumn{3}{|l|}{ Weight loss during last month } \\
\hline$>3 \mathrm{~kg}$ & 0 & $1 \cdot 9$ \\
\hline Does not know & 1 & $8 \cdot 6$ \\
\hline $1-3 \mathrm{~kg}$ & 2 & $22 \cdot 9$ \\
\hline No loss & 3 & $66 \cdot 6$ \\
\hline \multicolumn{3}{|l|}{ Mobility } \\
\hline Bed- or chair-bound & 0 & $1 \cdot 0$ \\
\hline Able to get out bed but does not go out & 1 & $3 \cdot 8$ \\
\hline Goes out & 2 & $95 \cdot 2$ \\
\hline \multicolumn{3}{|c|}{ Psychological stress/acute disease during past 3 months } \\
\hline Yes & 0 & $20 \cdot 0$ \\
\hline No & 2 & $80 \cdot 0$ \\
\hline \multicolumn{3}{|l|}{ Neuropsychological problems } \\
\hline Severe dementia or depression & 0 & $22 \cdot 9$ \\
\hline Mild dementia & 1 & $1 \cdot 0$ \\
\hline No psychological problems & 2 & $76 \cdot 2$ \\
\hline \multicolumn{3}{|l|}{ BMI $\left(\mathrm{kg} / \mathrm{m}^{2}\right)$} \\
\hline \multicolumn{3}{|l|}{ (a) MNA-TI } \\
\hline$<17$ & 0 & $3 \cdot 0 / 5 \cdot 3 *$ \\
\hline $17-19$ & 1 & $4 \cdot 5 / 2 \cdot 6$ \\
\hline $19-21$ & 2 & $10 \cdot 7 / 15 \cdot 8$ \\
\hline$>21$ & 3 & $81 \cdot 9 / 76 \cdot 3$ \\
\hline
\end{tabular}

(b) MNA-TII (This question was omitted)

Live independently ${ }^{\dagger}$

$\begin{array}{lll}\text { No } & 0 & 14 \cdot 3\end{array}$

$\begin{array}{lll}\text { Yes } & 1 & 85.7\end{array}$

Takes four or more kinds of prescribed drugs per day

Yes 0

No

Pressure sores or skin ulcers

Yes

No

ull meals daily

1

2

3

Daily consumption of protein-rich food

0 or 1 'yes'

2 'yes'

3 'yes'

Consumes $\geq 2$ servings of fruits or vegetables per day

No

Yes

Cups of water/fluid consumed

$<3$

$3-5$

$>5$

Mode of feeding

Unable to eat without assistance $\quad 0 \quad 1.0$

Self-fed with difficulty

Self-fed with any problem
Table 3 (Continued)

\begin{tabular}{|c|c|c|}
\hline Question & Score & $\%$ \\
\hline \multicolumn{3}{|l|}{ Self view of nutritional status } \\
\hline Views self as being malnourished & 0 & $13 \cdot 3$ \\
\hline Uncertain of nutritional status & 1 & $41 \cdot 9$ \\
\hline No nutritional problem & 2 & $44 \cdot 8$ \\
\hline \multicolumn{3}{|c|}{ Self view of health status compared to peers } \\
\hline Not as good & 0 & $9 \cdot 5$ \\
\hline Does not know & $0 \cdot 5$ & $36 \cdot 2$ \\
\hline As good & 1 & $41 \cdot 0$ \\
\hline Better & 2 & $13 \cdot 3$ \\
\hline \multicolumn{3}{|l|}{$\operatorname{MAC}(\mathrm{cm})$} \\
\hline \multicolumn{3}{|l|}{ (a) MNA-TI } \\
\hline$<22 \cdot 5 / 21 *$ & 0 & $1 \cdot 5 / 5 \cdot 3 *$ \\
\hline $22 \cdot 5-23 \cdot 5 / 21-22$ & $0 \cdot 5$ & $1 \cdot 5 / 0$ \\
\hline$>23 \cdot 5 / 22$ & 1 & $97 / 94 \cdot 7$ \\
\hline \multicolumn{3}{|l|}{ (b) MNA-TII } \\
\hline$<22 \cdot 5 / 21$ & 0 & $1 \cdot 5 / 5 \cdot 3$ \\
\hline $22 \cdot 5-23 \cdot 5 / 21-22$ & 1 & $1 \cdot 5 / 0$ \\
\hline$>23 \cdot 5 / 22$ & 2 & $97 / 94 \cdot 7$ \\
\hline \multicolumn{3}{|l|}{$\mathrm{CC}(\mathrm{cm})$} \\
\hline \multicolumn{3}{|l|}{ (a) MNA-TI } \\
\hline$<28 / 25^{*}$ & 0 & $1 \cdot 5 / 2 \cdot 6^{*}$ \\
\hline$>28 / 25$ & 1 & $98 \cdot 5 / 97 \cdot 4$ \\
\hline \multicolumn{3}{|l|}{ (b) MNA-TII } \\
\hline$<28 / 25$ & 0 & $1 \cdot 5 / 2 \cdot 6$ \\
\hline $28-29 / 25-26$ & 1 & $3 \cdot 0 / 0$ \\
\hline $29-30 / 26-27$ & 2 & $0 / 5 \cdot 3$ \\
\hline$\geq 30 / 27$ & 3 & $95 \cdot 5 / 92 \cdot 2$ \\
\hline
\end{tabular}

*Values for men and women, respectively.

†Subjects whose conditions were under good control and had rather normal daily life was considered able to live independently.

Compan et al. (1999) used the MNA to grade the nutritional status of 918 acute, sub-acute and long-term care older patients and found that malnutrition ranged from $25-33 \%$ among 13 subgroups including 88 neuropsychiatric patients whose mean MNA score was 21.1 (ranged from 16.7-21.7 among all subgroups).

Table 4 Distribution of nutritional status of mental patients according to the original and the modified MNA scales $(n=105)$

\begin{tabular}{llll}
\hline \multirow{2}{*}{ Version of MNA } & \multicolumn{3}{l}{ Nutritional status $(n, \%)$} \\
\cline { 2 - 4 } & Malnourished & At risk & Normal \\
\hline MNA-TI & $8(7 \cdot 6)$ & $23(21 \cdot 9)$ & $74(70 \cdot 5)$ \\
MNA-TII & $8(7 \cdot 6)$ & $21(20 \cdot 0)$ & $76(72 \cdot 4) *$ \\
\hline
\end{tabular}

*Analysis with Wilcoxon signed-rank test showed that the distributions of nutritional status assessed with the MNA-TI and MNA-TII were not significantly different from each other $(Z=-1.414$, $p>0 \cdot 05)$. All subjects identified malnourished or at risk of malnutrition by the MNA-TII were also identified as such by the MNA-TI. 
Table 5 Pearson's correlation $(r)$ of the total MNA scores with anthropometric, biochemical and health-related variables ${ }^{\dagger}(n=104)$

\begin{tabular}{|c|c|c|c|}
\hline Variable & MNA-TI & MNA-TII & Albumin \\
\hline Serum albumin $(\mathrm{g} / \mathrm{dl})$ & $0 \cdot 216^{*}$ & $0 \cdot 220 *$ & 1 \\
\hline BMI $\left(\mathrm{kg} / \mathrm{m}^{2}\right)$ & $0 \cdot 520 * * *$ & $0.468 * * *$ & $0 \cdot 136$ \\
\hline \multicolumn{4}{|l|}{$\operatorname{MAC}(\mathrm{cm})$} \\
\hline Men & $0 \cdot 566 * *$ & $0 \cdot 547 * * *$ & $0 \cdot 266^{*}$ \\
\hline Women & $0 \cdot 358 *$ & $0 \cdot 329 *$ & $-0 \cdot 051$ \\
\hline \multicolumn{4}{|l|}{$\mathrm{CC}(\mathrm{cm})$} \\
\hline Men & $0 \cdot 448 * * *$ & $0 \cdot 465 * * *$ & $0 \cdot 172$ \\
\hline Women & $0.436 * *$ & $0 \cdot 443 * *$ & $-0 \cdot 117$ \\
\hline No. of prescribed medicine & $-0 \cdot 348 * * *$ & $-0 \cdot 370 * *$ & $-0 \cdot 330 * *$ \\
\hline Appetite status ${ }^{*}$ & $0.656 * *$ & $0.638 * * *$ & $0 \cdot 032$ \\
\hline$\%$ weight change & $-0.464 * * *$ & $-0.425^{* * *}$ & $-0 \cdot 084$ \\
\hline
\end{tabular}

$* p<0.05, * p<0.01, * * p<0.001$.

${ }^{\dagger}$ No significant correlations were observed with $\mathrm{W} / \mathrm{H}$, haematocrit, serum cholesterol and years of mental disorder.

*Self-rated with a five-level Likert type scale ranging from very poor to very good.

\section{Serum albumin}

Low serum albumin and unintended body weight loss are the two indicators often used to suggest deteriorating nutritional conditions in neuropsychiatric patients in Taiwan. However, according to results of the present study, the level of serum albumin does not correlate well with the MNA scores. Neither does it correlate well with most of the major general nutrition indicators such as BMI, CC, appetite status and weight loss. The possible explanation for the lack of correlation may be due to the fact that (1) most patients in this study are mature adults younger than 65 years in age, (2) only relatively few $(7 \cdot 6 \%)$ of these patients have serum albumin below $3.5 \mathrm{~g} / \mathrm{dl}$ (the average is $4.1 \mathrm{~g} / \mathrm{dl}$ ), and (3) serum albumin has a relatively long turnover time (half-life 14-20 days) and thus is relatively slow in responding to nutritional changes (Lee \& Nieman 2003b). Additionally, some drugs taken by the patients may confound serum albumin concentrations by affecting their appetite or liver function. Thus, although albumin is a valuable indicator of chronic protein-calorie malnutrition in young children and older adults, it does not appear to be a sensitive nutritional indicator for adult neuropsychiatric patients.

\section{Correlation of MNA scores with other parameters}

Among the variables examined, appetite status, BMI, MAC and CC (in men), \% weight change during the past three months and number of prescribed drugs showed strong correlations with the total MNA scores, CC and MAC (in women) showed moderate correlations whereas serum albumin showed weak but significant correlations. The correlation of each of the above-mentioned variables with serum albumin is much weaker than that with the MNA scores. These results further suggest that the MNA is a valuable and effective tool for identifying individuals at risk of malnutrition among neuropsychiatric patients.

\section{Limitations to the study}

Patients with neuropsychiatric disorders have a wide range of potential nutritional abnormalities. Depending on the nature of the disorder, nutritional disorders may include increased weight gain (due to over-eating), delusions, catatonic behaviour, anorexia, drug-nutrient interactions, nutrient imbalance and refusal to eat or drink. The MNA is mainly for identifying individuals with general malnourishment or at risk of malnutrition. It would have limited ability in pinpointing other nutritional problems associated with neuropsychiatric disorders. More elaborate assessments should be performed to determine eating habits and food beliefs, or possible drug-nutrient interactions. Also, results of this study only represent the average condition of the participating subjects in this study. Neuropsychiatric disorders have diverse causes and complex nutritional problems. Without adequate number of subjects in some of the subtypes, the current study is unable to assess the functionality of the MNA in each subtype of the disorder.

It should also be reminded that subjects interviewed in this study were cognitively 'functional' individuals. For those who have impaired cognition, their nutritional status would probably be worse. Further, while MNA might be of value in identifying individual at risk of malnutrition, it would not totally replace well-trained care-givers. Close monitoring and observation of patients' eating behaviour, in addition to periodic nutritional assessment, is essential for early detection of emerging nutritional problems.

\section{Relevance to clinical practice}

The MNA-TII, being non-invasive and without the BMI question, does have an advantage over the original MNA which requires weight and height data for the calculation of BMI. Such an advantage does have practical implications in the care practice of neuropsychiatric patients. Measuring weight and height is not always easy or possible, especially for those who are bed-ridden. Some neuropsychiatric patients may also refuse to be weighed. Thus, the MNA-TII has the potential to improve the professional efficiency of the primary care workers by making patients' periodic assessment of nutritional status a less demanding task. 


\section{Conclusions}

The development of malnutrition is a continuum and often is unnoticeable, especially in mature adults. Malnutrition starts with inadequate or imbalanced food intakes followed by changes in biochemical indicators and possibly also body composition. Neuropsychiatric patients are at extra risk of having malnutrition due to their unusual dietary habits or belief, drug effects or altered lifestyles. Nutritional status and mental status can interact with each other bidirectionally. It is important that the assessment of their nutritional status be conducted routinely so that emerging nutritional problems is detected and intervened before progressing to become a major health problem. The MNA, especially in its modified version (without BMI) appears to serve this purpose well.

\section{Acknowledgements}

This study was supported by a grant-in-aid provided by the Bureau of Nursing and Health Services Development of the Department of Health of Taiwan. The authors wish to thank the hospital for permitting the interviews, the care workers for assisting in the interview process and the participants for their cooperation during the course of this study.

\section{Contributions}

Study design: ACT, S-FT; data collection and analysis: YTC, TLC, S-NC and manuscript preparation: ACT, TLC.

\section{References}

Abayomi J \& Hackett A (2004) Assessment of malnutrition in mental health clients: nurses' judegement vs. a nutritional risk tool. Journal of Advanced Nursing 45, 430-437.

American Psychiatric Association (2000) Diagnostic and Statistical Manual of Mental Disorders, 4th edn. Text Revision (DSM-IVTR). American Psychiatric Association, Washington DC.

Asplund KJ, Normark M \& Pettersson V (1981) Nutritional assessment of psychogeriatric patents. Age and Ageing 10, 87-94.

Brown S, Birtwistle J, Roe L \& Thompson C (1999) The unhealthy lifestyle of people with schizophrenia. Psychological Medicine 29, 697-701.

Compan B, Di Castri A, Plaze JM \& Arnaud-Battandier F (1999) Epidemiological study of malnutrition in elderly patient in acute, sub-acute and long-term care using the MNA. The Journal of Nutrition, Health \& Aging 3, 146-151.

Coppen A \& Bolander-Gouaille C (2005) Treatment of depression: time to consider folic acid and vitamin $\mathrm{B}_{12}$. Journal of Psychopharmacology 19, 59-65.

Gariballa S \& Forster S (2007) Effects of dietary supplements on depressive symptoms in older patients: a randomized double-blind placebo-controlled trial. Clinical Nutrition 26, 545-551.
Gray GE \& Gray LK (1989) Nutritional aspects of psychiatric disorders. Journal of the American Dietetic Association 89, 14921497.

Guigoz Y, Vellas BJ \& Garry PJ (1994) The Mini Nutritional Assessment (MNA): a practical assessment tool for grading the nutritional state of elderly patients. in Facts and Research in Gerontology (Vellas BJ, Guigoz Y, Garry PJ \& Albarede JL eds). Serdi Publishing Co., New York, NU. 1994 (Supplement: Nutrition), pp. 15-60.

Guigoz Y, Lauque S \& Villas BJ (2002) Identifying the elderly at risk for malnutrition. The Mini Nutritional Assessment. Clinics in Geriatric Medicine 18, 737-757.

Kerperman RFJ, Veurink M, van der Wal T, Knegtering H, Bruggeman R, Fokkema MR, Kema IP, Korf J \& Muskiet FAJ (2006) Low essential fatty acid and B-vitamin status in a subgroup of patients with schizophrenia and its response to dietary supplementation. Prostaglandins, Leukotrienes and Essential Fatty Acids $74,75-85$.

Lee RD \& Nieman DC (2003a) Chapter 7: Assessment of the hospitalized patient. In Nutritional Assessment, 3rd edn (Lee RD \& Nieman DC eds). McGraw-Hill, New York, NY, pp. 216-250.

Lee RD \& Nieman DC (2003b) Chapter 9 Biochemical Assessment of Nutritional Status. In Nutritional Assessment, 3rd edn. McGraw Hill, New York, NY. pp. 303-336.

McCreadie RG (2003) Diet, smoking and cardiovascular risk in people with schizophrenia. Descriptive study. British Journal of Psychiatry 183, 534-539.

Molteno C, Smit I, Mills J \& Huskisson J (2000) Nutritional status of patients in a long-stay hospital for people with mental handicap. South African Medical Journal 90, 1135-1140.

Prince M, Patel V, Saxena S, Maj M, Maselko J, Phillips MR \& Rahman A (2007) No health without mental health. Lancet 370, 859-877.

Saxena S, Jane-Llopis E \& Hosman C (2006) Prevention of mental behavioural disorders: implications for policy and practice. World Psychiatry 5, 5-14.

Sullivan A, Diet D \& Tucker R (1999) Meeting the nutritional needs of people with mental health problems. Nursing Standard 13, $11-17$.

Tsai AC \& Ku PY (2008) Population-specific Mini Nutritional Assessment effectively predicts the nutritional state and follow-up mortality of institutionalized elderly Taiwanese regardless of cognitive status. British Journal of Nutrition 100, 152-158.

Tsai AC \& Shih CL (2009) A population-specific Mini Nutritional Assessment can effectively grade the nutritional status of stroke rehabilitation patients in Taiwan. Journal of Clinical Nursing 18, 82-88.

Tsai AC, Ho CS \& Chang MC (2007a) Population-specific anthropometric cut-points improve the functionality of the Mini Nutritional Assessment (MNA) in elderly Taiwanese. Asia Pacific Journal of Clinical Nutrition 16, 656-662.

Tsai AC, Ho CS \& Chang MC (2007b) Assessing the prevalence of malnutrition with the Mini Nutritional Assessment (MNA) in a nationally representative sample of elderly Taiwanese. Journal of Nutrition, Health \& Aging 14, 239-243.

Tsai AC, Ku PY \& Tsai JD (2009) Population-specific anthropometric cutoff standards improve the functionality of the Mini Nutritional Assessment without BMI in institutionalized elderly in Taiwan. Journal of Nutrition, Health \& Aging (in press). 